\title{
Femtochemistry: The Role of Alignment and Orientation
}

\author{
Ahmed H. Zewail $\dagger$ \\ Arthur Amos Noyes Laboratory of Chemical Physics, $\ddagger$ California Institute of Technology, \\ Pasadena, California 91125, U.S.A.
}

\begin{abstract}
Some aspects of alignment and orientation have been considered for femtochemistry experiments. Elementary theoretical descriptions of the time evolution of alignment and angular momenta have been discussed and related to the radial and angular parts of the potential-energy surface. Applications to ICN unimolecular dissociation, $\mathrm{H}+\mathrm{CO}_{2}$ 'oriented' bimolecular reaction, and crossings between different potential-energy curves in alkali-metal halide reactions $(M+X)$ are given. Emphasis is on the femtosecond dynamics of the transition-state region and the clocking of fragment separation in real time.
\end{abstract}

\section{Introduction}

In femtochemistry, chemistry on the femtosecond timescale, one monitors the reaction using two laser pulses of different colours. The first pulse initiates the reaction and sets the zero of time (clocking). The second pulse probes the fragments as they separate from each other or as they form new bonds. Because of the time resolution and sensitivity, the separation can be viewed with sub-ångstrom resolution. Elsewhere, ${ }^{1-7}$ the concept of the experiments has been discussed in relation to the potential-energy surfaces (PES) of the reaction, and applications to a number of reactions have been detailed. Here, we shall address the role of alignment and orientation in these femtochemical experiments.

On the femtosecond timescale, the fragments are in close proximity and the time evolution of coherence, angular momenta and possibly trapping resonances is expected to be important. Hence, borrowing from techniques used to study alignment of fragments at asymptotic distance separations ${ }^{8}$ (i.e. time $\rightarrow \infty$ ) one can perhaps observe the 'during' alignment of the reaction if femtosecond pulses are used. These studies should help in answering some new questions about the ephemeral transition-states orientation and population, and the angular dependence of the PES.

Some elementary theoretical descriptions of these time-dependent processes are given here. Comparison with experiments involving dissociation reactions will be made and extension to 'oriented' bimolecular reactions (collision complexes) will be discussed. We will first briefly highlight the experimental methodology and results.

\section{The FTS Technique and Some Applications}

The main technique described here is femtosecond transition-state spectroscopy (FTS) which is a pump-probe method that allows us to obtain snapshots of reactions while fragments are separating, or encountering each other. If ultrashort pulses are employed, the sensitivity of the detection is enhanced by a factor of $10^{4}-10^{6}$ when compared with time-integrated, steady-state experiments. Essentially all molecules traversing the transition states can be detected, and this is a key feature of femtochemistry. The difficulty,

$\uparrow$ John Simon Guggenheim Foundation Fellow.

$\ddagger$ Contribution no. 7844 . 
however, comes in generating pulses and in developing the techniques for observing changes on the femtosecond timescale.

The FTS signal recorded will be some measure of the absorption by the fragment of the probe pulse at wavelength $\lambda_{2}$ as a function of the time-delay $\tau$ between pump and probe. This could be accomplished, for instance, by measuring the change in the transmission of the probe. Since the experiment would not be background-free, far better sensitivity can be obtained through, for instance, the detection of the laser-induced fluorescence (LIF), or the multiphoton ionization (MPI) generated by the probe. Typically, we measure the FTS signal when $\lambda_{2}$ is tuned to the absorption wavelength of the free fragment, $\lambda_{2}^{\infty}$ (where $\infty$ denotes that $R \rightarrow \infty$ ), and at a number of wavelengths absorbed by the transition states, $\lambda_{2}^{*}$ (where ${ }^{*}$ denotes the transition-state region of $R$ ). Then a surface of measurements $A\left(t ; \lambda_{2(a)}^{*}, \lambda_{2(b)}^{*}, \ldots, \lambda_{2}^{\infty}\right)$ is constructed. This surface is related to the dynamics and to the PES of the reaction, as we will discuss below. The experiments are then repeated for different pump wavelengths $\left(\lambda_{1}\right)$.

\section{(a) Unimolecular Reactions}

In the simplest FTS experiment (clocking), the probe is centred at an absorption of one of the free fragments. In this case, the absorption of the probe will initially be negligible and will only become substantial when the fragments achieve large internuclear distance and are therefore (essentially) non-interacting. The delay at which the probe absorption 'turns on,' $\tau_{1 / 2}$, is therefore a direct measurement of the time required for complete (or nearly complete) internuclear separation. In other words, FTS provides a 'clock' of the time required to break the bond, and thus is a fundamental measurement.

The experiment can be pictured simply as a measure of the femtosecond 'time-offlight' on the excited-state PES from the initially excited configuration at $R_{0}$ to the free fragment configuration, where the probe opens a 'window' on the PES. If the experiment is performed for various probe spectral widths, then additional information on the asymptotic shape of the potential can be obtained. Additionally, the clocking experiment offers significant practical advantages which have been discussed by Rosker et al. ${ }^{3}$ Such clocking experiments have been performed on the dissociation reaction of ICN,

$$
\mathrm{ICN}^{*} \rightarrow[\mathrm{I} \cdots \mathrm{CN}]^{* *} \rightarrow \mathrm{I}+\mathrm{CN}
$$

and provide $\tau_{1 / 2}=205 \pm 30 \mathrm{fs}$ (see fig. 1 ).

FTS experiments can also be made by tuning $\lambda_{2}$ away from the transition of the free fragment. The final products will thus not absorb the probe wavelength, but instead the transition states of the reaction may do so. The FTS transient will therefore be expected to build up, as the molecules enter the optically coupled region of the probe, and then subsequently decay, as the molecules move on to final products (see fig. 2). By obtaining FTS transients for various values of $\lambda_{2}^{*}$, information on the shapes of the PESs involved can be obtained. These experiments have been detailed elsewhere by Roster et al. ${ }^{3}$ and discussed in relation to the PESs.

To relate these measurements to a dissociation time and to the characteristics of the PES, we first consider for repulsion between the I and the $\mathrm{CN}$ fragments a potential of the form: ${ }^{9}$

$$
V(R, \theta)=V_{0} \exp \left(-R / L_{1}\right) f(R, \theta)
$$

where $L_{1}$ is the repulsion length parameter and $f(R, \theta)$ describes the angular part. The classical equation of motion for this system, without $f(R, \theta)$, can be integrated to give analytically the internuclear separation of the fragments as a function of time, $R(t)$, and the corresponding potential as a function of time, $V(t)$.

We then ask, what defines a dissociation time? If it were defined as the time for the two fragments to reach a separation at which their interaction energy is negligible, the dissociation time would depend on the sensitivity with which the energy can be measured; 

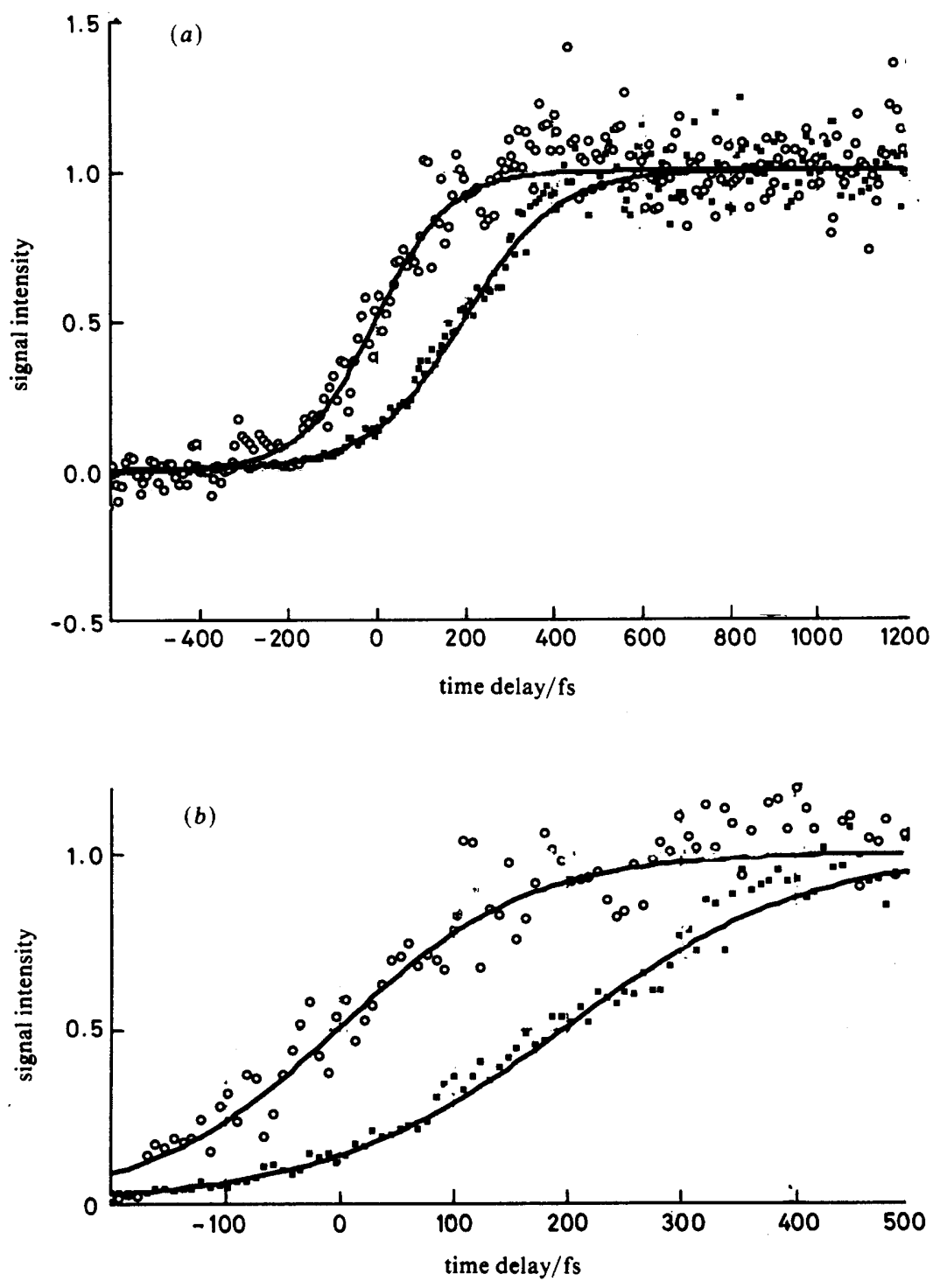

Fig. 1. Typical on-resonant FTS results for the ICN reaction. (a) $\bigcirc$, the MPI calibration signal, which gives the detection-response function and the zero of time; ${ }^{3} \boldsymbol{\square}$, the FTS data for ICN, taken with $\lambda_{2}=\lambda_{2}^{\infty}$. Note the time delay between these traces, $\tau_{1 / 2} .(b)$ The same data,shown on an expanded scale.

an infinite time is required to reach infinite separation. A more invariant definition is obtained as follows: consider the difference between the time taken for the fragments to separate from $R_{0}$, the separation at the instant of photoexcitation, to $R$ (as they travel along the actual potential) minus the time which would be required had the fragments travelled at the terminal velocity $(v)$. Then $\tau_{d}$ is defined as the limit of this difference as $R \rightarrow \infty$. For the potential described above (with no anisotropy), one obtains: $\tau_{\mathrm{d}}=\left(L_{1} / v\right) \ln 4$. While it is well defined, this $\tau_{\mathrm{d}}$ is obtainable only from a 


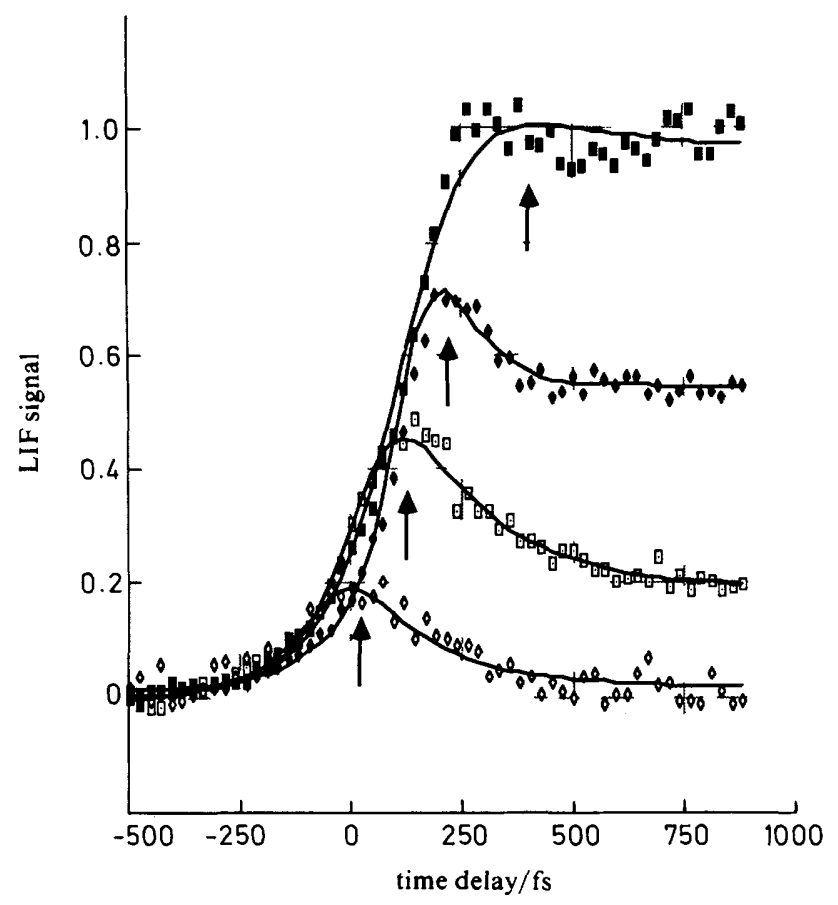

Fig. 2. Typical off-resonant FTS results for the ICN reaction. $\square, \lambda_{2}=389.7 \mathrm{~nm} ; \bullet, \lambda_{2}=389.9 \mathrm{~nm}$; $\square, \lambda=390.4 \mathrm{~nm} ; \diamond, \lambda_{2}=391.4 \mathrm{~nm}$ The zero of time is determined separately for each data set.

Comparison with theory is discussed in ref. (3).

hypothetical experiment requiring the measurement of minute differences in long arrival times (see fig. 3).

A more useful description of bond breaking has been recently developed ${ }^{3,10}$ to consider the time dependence of fragment separation and the absorption by fragments during this separation. When the fragments develop to be spectroscopically free, the absorption achieves a maximum. Dissociation can be defined as the time delay when the mid-point of the absorption is reached:

$$
\tau_{1 / 2}=\left(L_{1} / v\right) \ln (4 E / \gamma) \text {. }
$$

Here, $\gamma$ is the half-width of the energy distribution of the probe pulse and $E$ is the energy available above dissociation $(E \gg \gamma)$. An important observation is contained within this analysis: the probe spectral width determines a 'window' through which the free $\mathrm{CN}$ is viewed, and so affects $\tau_{1 / 2}$. Since we have both $\gamma\left(40 \mathrm{~cm}^{-1}\right)$ and $E\left(6000 \mathrm{~cm}^{-1}\right)$, the above expression yields for the repulsion length parameter $L_{1}=0.8 \AA$, for the exponential potential discussed above. A similar procedure can be used to calculate length parameters of other potential shapes, e.g. $R^{-n}$. According to the above analysis, the potential falls to 1 /e of its initial value in $0.8 \AA$, but the distance corresponding to $\tau_{1 / 2}$ (the time taken for the potential to drop to $\gamma$ ) is $4 \AA$.

There are several points to be made regarding this reaction. First, the PES of ICN dissociation is more complex than that illustrated here because there are two channels (with possible crossings) for dissociation involving the production of iodine either in its ground or its excited spin-orbit states (fig. 4). However, at $306 \mathrm{~nm}$ our available energy is just below the excited $I^{*}$ state channel, and it is known experimentally that at this excitation wavelength the reaction is to the ground-state channel, which correlates 


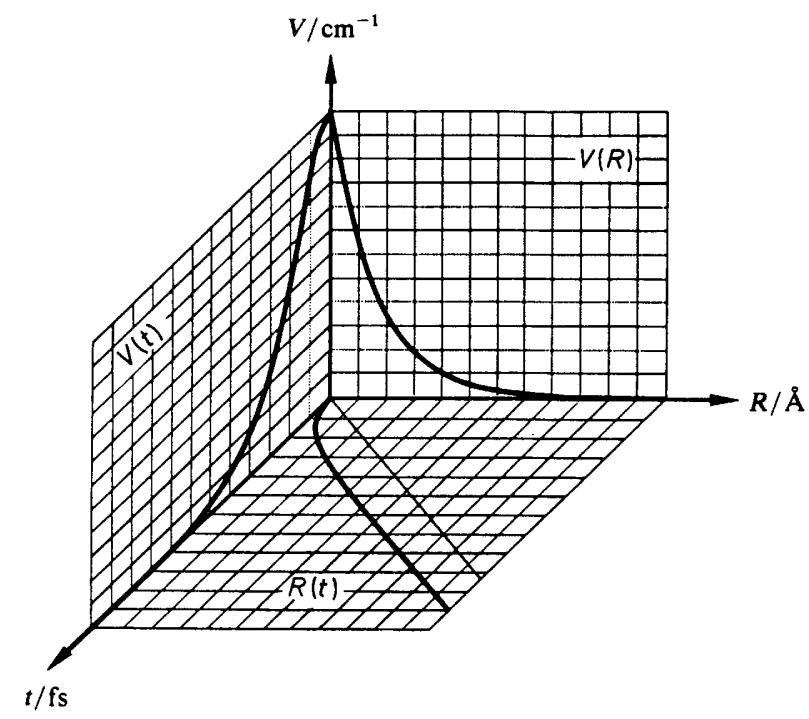

Fig. 3. A potential-energy surface as a function of the parameter $t$ (time), with $25 \mathrm{fs}$ per division, and as a function of the parameter $R$ (internuclear distance), with $0.5 \AA$ per division. An exponential shape is assumed and the initial potential energy at $t=0$ and $(R=0)$ is $6000 \mathrm{~cm}^{-1}$ $\left(17.2 \mathrm{kcal} \mathrm{mol}^{-1}\right.$ above dissociation). Note that the recoil velocity, given by the instantaneous slope of $R(t) v s$. $t$, rapidly achieves the terminal value. The difference between $R(t)$ and the straight line (which is determined by the terminal velocity) approaches a constant at long time; this difference represents the invariant $\tau_{\mathrm{d}}$ defined in the text.

with a bent state. The measurements reported here clock the dynamics of $\mathrm{I}-\mathrm{CN}$ recoil and the development of free CN. As pointed out by several authors, ${ }^{9,11,12}$ the dynamics that produce rotational excitation are due to bending, and could be on a shorter timescale. Time-resolved alignment experiments (discussed below) should aid us in resolving these bending dynamics. At our wavelength of excitation, the product rotational-state distribution of the nascent $\mathrm{CN}$ was explained by nearest-neighbour repulsion effects exclusively. ${ }^{13}$ This is consistent with the picture here of repulsion between $\mathrm{CN}$ and iodine. Secondly, the absorption measurement depends also on the characteristics of the upperstate $\left(\mathrm{I}+\mathrm{CN}^{*}\right)$ potential. However, in the asymptotic limit $(R \rightarrow \infty), \tau_{1 / 2}$ is described, for the potential given above, by eqn (3), and as discussed elsewhere, ${ }^{3,14}$ from the shape of the transients, the direction of the probe wavelength shifts and the effect of tuning the pump wavelength can separate $V_{1}$ and $V_{2}$ dynamics. Finally, we assumed here a repulsive potential, but there exists a method to invert the data to give the shape of the PES and to account for the attractive terms in the potential. ${ }^{15}$ What about the angular part $f(R, \theta)$ of the PES? This we address below in section 3 .

Theoretically, these observations are reproduced using classical-mechanical ${ }^{3}$ as well as quantum-mechanical ${ }^{16}$ treatment of the dissociation (see fig. 5). Even kinetics can describe the general behaviour of the transients as shown in ref. (3). From these results we determine that the transition states en route to dissociation live for only $50-20 \mathrm{fs}$, depending on the $R$ region probed.

\section{(b) Extension to Bimolecular Reactions}

An important new issue arises for bimolecular reactions that is not a concern in the unimolecular case. For a unimolecular reaction, the reaction begins when the laser 


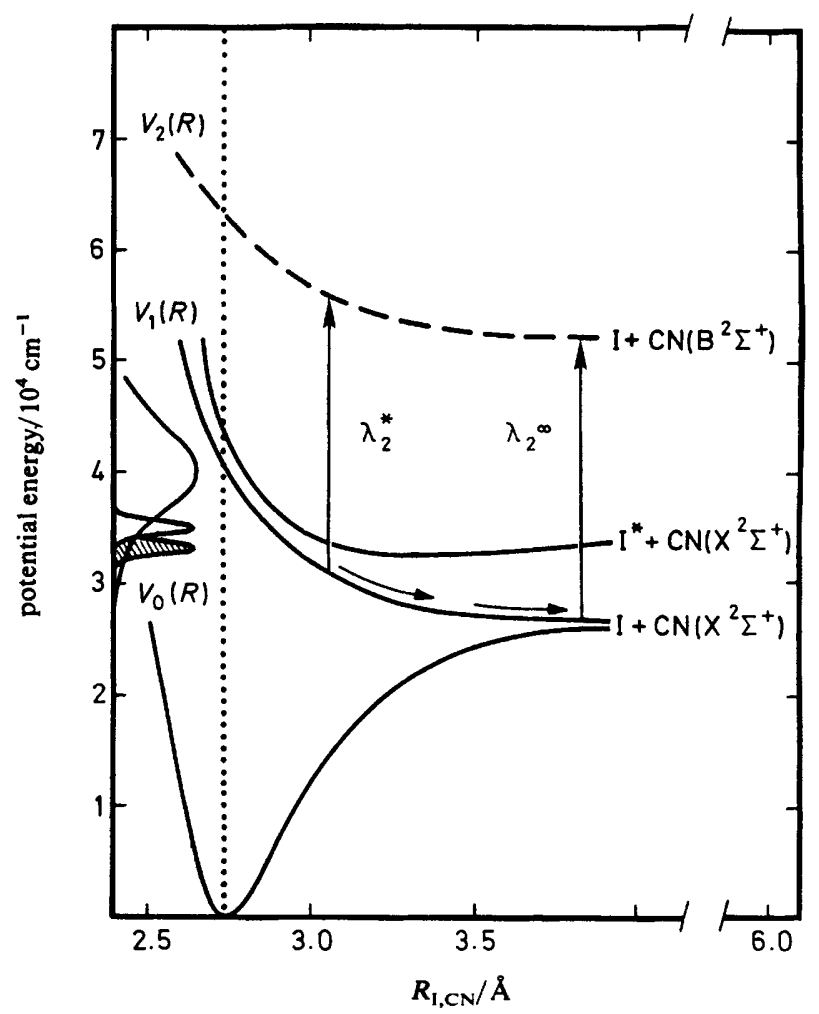

Fig. 4. The (typical) potential-energy surfaces of ICN and the concept of FTS experiments at $\lambda_{2}^{*}$ and $\lambda_{2}^{\infty}$. The pump pulse causes a vertical transition from $V_{0}$ to $V_{1}$, and the subsequent motion on this surface is indicated. The transition state is probed by $\lambda_{2}^{*}$, or the final state by $\lambda_{2}^{\infty}$, which are shown by the respective arrows from $V_{1}$ to $V_{2}$. The absorption spectrum of ICN and the pump energies used are sketched on the left. Note that the difference between $\lambda_{2}^{*}$ and $\lambda_{2}^{\infty}$ is not to scale and that the van der Waals wells are not shown.

pulse initiates excitation and subsequent dissociation. Thus, the interval between the firing of this laser pulse and the subsequent probe laser pulse represents the actual amount of time that the reaction has been taking place. However, for a bimolecular reaction, whether in bulk or in crossed beams, there is no comparable way to establish the beginning point for the reaction. So there would appear to be no hope of determining the time of formation and decay of transient collision complexes with lifetimes of only femtoseconds to picoseconds.

However, a special trick enables one to establish the zero of time within an uncertainty governed by the laser pulse duration only, for a whole class of bimolecular reactions. This has now made it possible to study real-time dynamics of these bimolecular reactions. The first of these experiments by Scherer et al., ${ }^{17}$ in collaboration with Bernstein, has been conducted in the picosecond, rather than femtosecond, time domain, but the principle is the same. The method involves the use of a beam of a van der Waals 'precursor molecule' and containing the potential reagents in close proximity. Here, we make use of the approach of weak molecular complexes, as pioneered by the groups of Wittig and Soep to study product-state distributions, ${ }^{18,19}$ so we can establish the zero of time and hence follow the course of the reaction in real time. For the reaction studied,

$$
\mathrm{H}+\mathrm{OCO} \rightarrow[\mathrm{HOCO}]^{\ddagger} \rightarrow \mathrm{OH}+\mathrm{CO}
$$




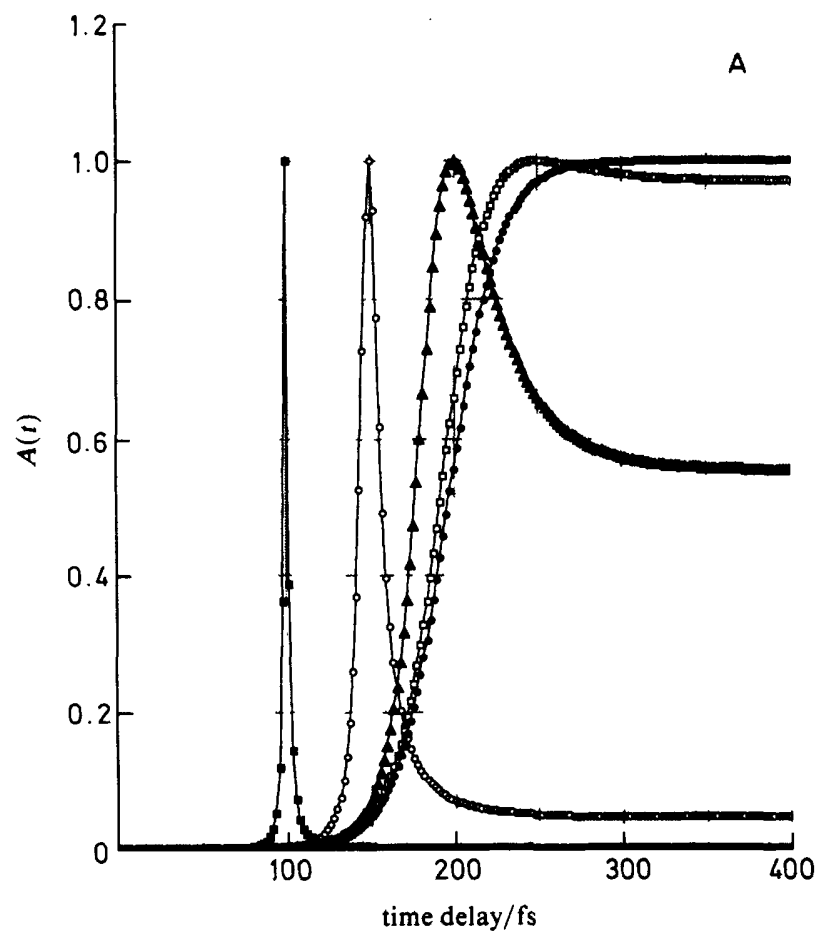

Fig. 5. (A) Predicted FTS results from classical mechanical calculation (see text): parameters are $L_{1}=0.8 \AA, v=0.026 \AA \mathrm{fs}^{-1}, E=6000 \mathrm{~cm}^{-1}$, and $\gamma=40 \mathrm{~cm}^{-1}$ (representative for ICN). The curves shown are for (from left-to-right) $t^{*}=100,150,200,250$ and $1000 \mathrm{fs}$. The peak amplitude of each data set has been normalized to one and the calculation does not take into account the finite temporal width of the pulses. [For more details see ref. (3).] Note the delay of the free fragment absorption, by $200 \mathrm{fs}$, and the build-up and decay of absorption of fragments in the transition region $\left(\lambda_{2}^{*}\right)$.

the precursor molecule was $\mathrm{IH} \cdots$ OCO, formed in a free-jet expansion of a mixture of $\mathrm{HI}$ and $\mathrm{CO}_{2}$ in an excess of helium carrier gas. Such van der Waals complexes have favourable geometry which limits the range of impact parameters and angles of attack of the $\mathrm{H}$ on the OCO. ${ }^{18}$

To clock the reaction, an ultrashort laser pulse initiates the experiment by photodissociating the $\mathrm{HI}$, ejecting a translationally hot $\mathrm{H}$ atom in the general direction of the nearest-neighbour $\mathrm{O}$ atom in the $\mathrm{CO}_{2}$. A probe laser pulse tuned to a wavelength suitable for detection of the $\mathrm{OH}$ was delayed relative to the photolysis pulse using a Michelson interferometer. The $\mathrm{OH}$ appeared after a delay of a few picoseconds from the initiation pulse. This delay represents a direct measure of the lifetime of the $[\mathrm{HOCO}]^{\ddagger}$ collision complex at the available energy of the collision. The lifetime changes with translational energy. A new series of experiments using femtosecond pulses is being undertaken in order to follow the formation and decay of the collision complex in the femtosecond region, and to study the effect of limited impact parameter (orientation), an important feature of this approach, on the reaction dynamics. Comparison with classical trajectory calculations, ${ }^{20}$ based upon the ab initio calculated PES, will be important.

It is probable that this technique will be applicable to a wide variety of bimolecular reactions, including not only those involving long-lived collision complexes that last for 

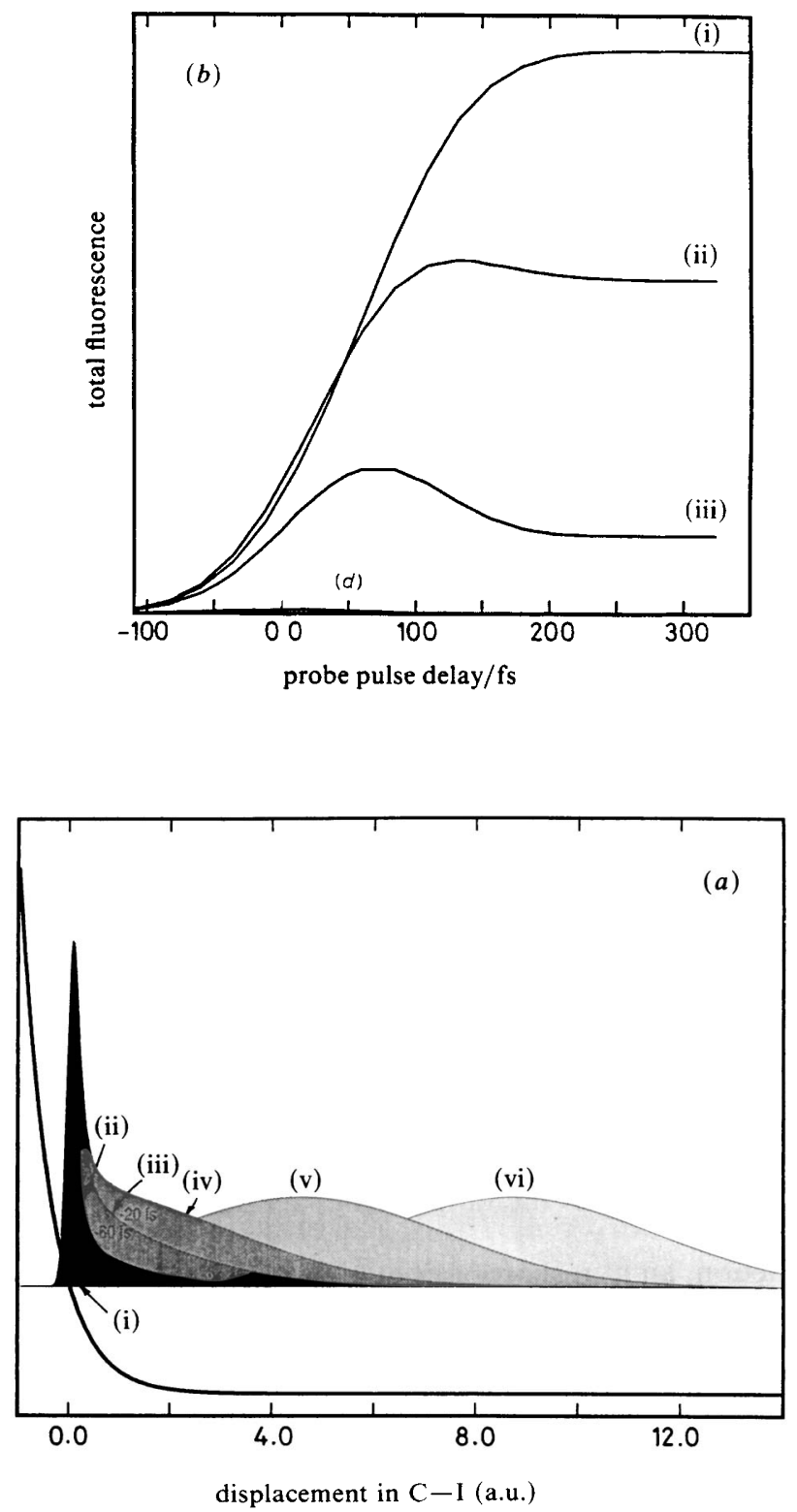

Fig. 5. (B) Predicted FTS results (b) from quantum calculation of the wavepacket propagation (a) for the ICN dissociation, using $125 \mathrm{fs}$ pulses. The general agreement with experiment and with the classical model is good. Taken from ref. (16). (a) (i) -140 , (ii) -60 , (iii) -20 , (iv) 20 ,

(v) 100 and (vi) $180 \mathrm{fs}$; (b) (i) 388.9 , (ii) 389.5 and (iii) $390.5 \mathrm{~nm}$. 


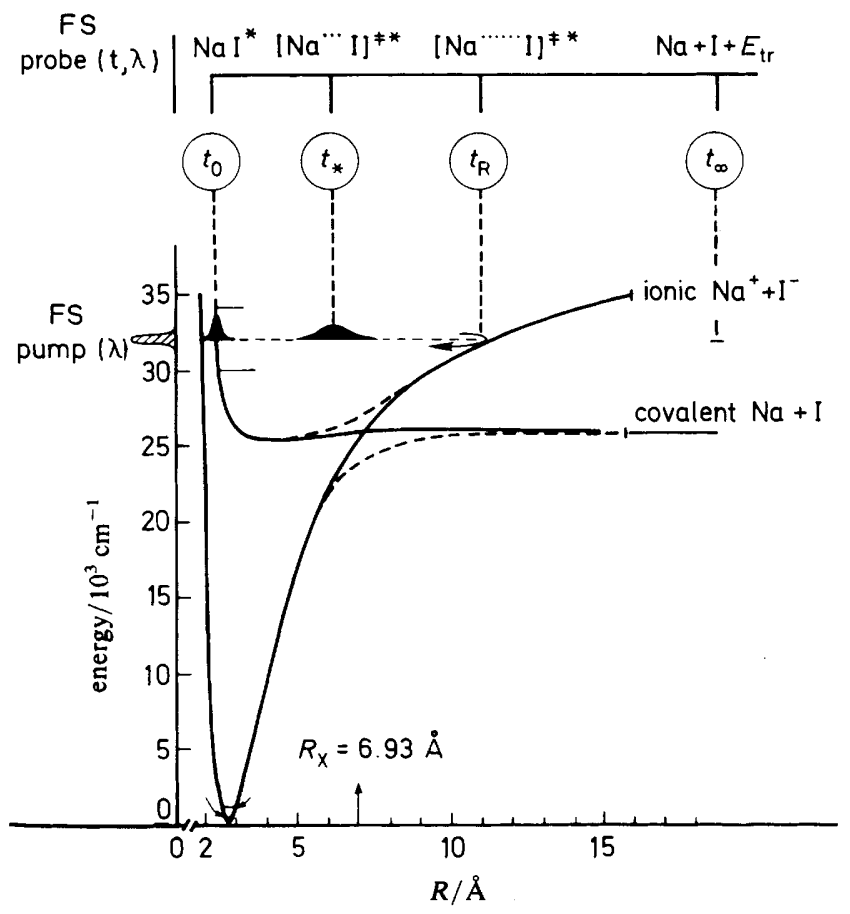

Fig. 6. The PES of an alkali-metal halide ( $\mathrm{NaI})$ reaction, and the concept of FTS experiments at $\lambda_{2}^{\infty}$ and $\lambda_{2}^{*}$.

picoseconds, but also direct-mode reactions with transition-state lifetimes in the femtosecond range. As with unimolecular reactions, off-resonance detection should allow detailed measurements of the build-up and decay of the transition states with the zero of time for the bimolecular reaction being known to fs time resolution. The fact that the reagents are oriented (at least to some extent) also makes the alignment experiment described below of particular interest.

\section{(c) Reactions from the 'Alkali Age': Covalent to Ionic Crossings}

The femtochemistry discussed thus far for unimolecular elementary reactions [section $2(a)$ ] involves the process of bond breaking on dissociative potential-energy surfaces. The molecules are in 'transition' for only $50 \mathrm{fs}$ or so, as evidenced by the rise and decay observed in the ICN experiments and confirmed by theory. If, however, in the process of falling apart, the system encounters a well in the potential-energy surface, or if there is more than one degree of freedom involved, the system can be 'trapped' and thus exhibit behaviour indicative of quasi-bound states, or resonances. In real time, manifestation of these resonances would be a slow-down in the appearance of free fragments and possibly the appearance of oscillations reflecting the vibrational resonance frequency of the wavepacket of the dissociating fragments.

The classic reactions of alkali-metal-halogen are prototype systems for such studies. This class of reactions has been studied before in the 'alkali age' of molecular-beam scattering of alkali metal $(M)$ and halogen $(X),{ }^{21-24}$ and observing their real-time behaviour will be of great interest to current studies in the 'femto age.' Because of the ionization potential of $M$ and the electron affinity of $X$, the energy of $M^{+}+X^{-}$is higher than that of $\mathbf{M}+\mathrm{X}$. In the ground state the molecule is ionic $\mathbf{M}^{+} \mathbf{X}^{-}$, and this state correlates with $\mathrm{M}^{+}+\mathrm{X}^{-}$(see fig. 6 ). Hence, the covalent potential-energy curve $(M+X)$ 


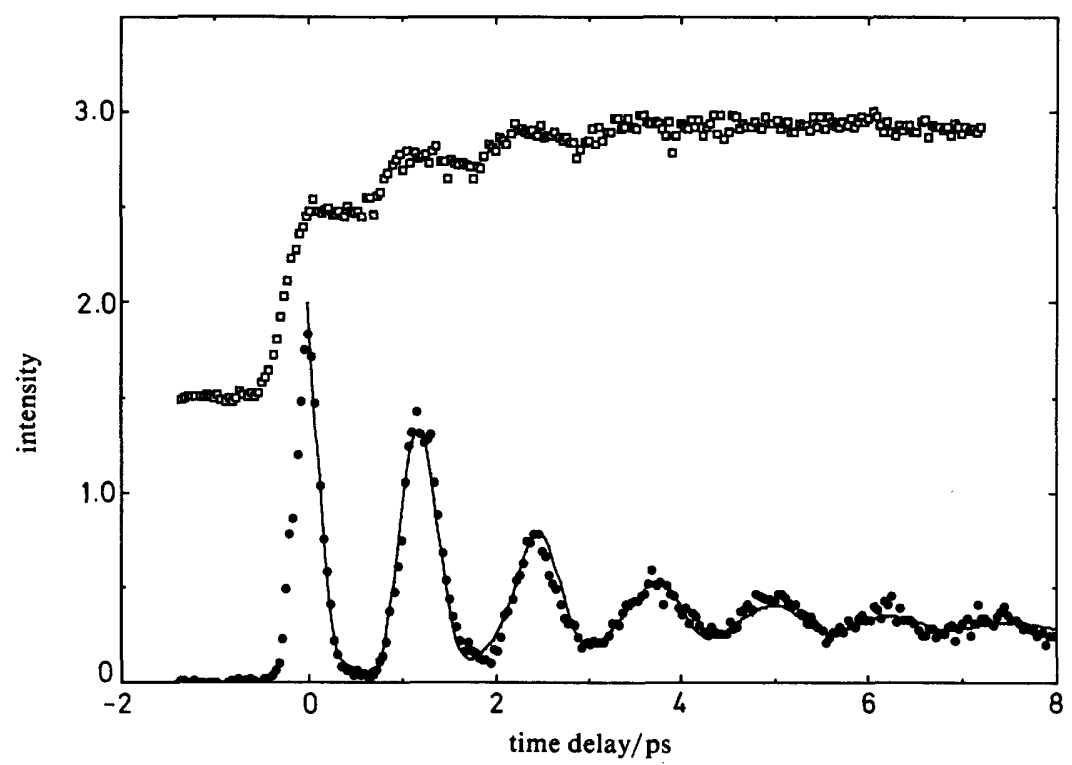

Fig. 7. Experimental FTS results obtained at $\lambda_{2}^{*}$ and $\lambda_{2}^{\infty}(589 \mathrm{~nm})$ for the reaction: $\mathrm{Na}$ $\mathrm{I}^{*} \rightarrow[\mathrm{Na} \cdots \mathrm{I}]^{\ddagger *} \rightarrow \mathrm{Na}+\mathrm{I}$. The oscillatory (resonance) behaviour at the bottom is obtained $\left(\lambda_{1}=310 \mathrm{~nm}\right)$ when probing at $\lambda_{2}^{*}$, while the transient at the top was obtained by probing the free fragment $(\mathrm{Na})$. Note that when probing the free fragment the signal shows an 'integration' which results in plateaux separated by exactly the resonance frequency. The $\mathrm{NaBr}$ reaction was also studied and results are discussed in ref. (4) and (5).

crosses the ionic (Coulombic) curve. Because of this crossing (or avoided crossing) at certain $\mathrm{M} \cdots \mathrm{X}$ separation $\left(R_{\mathrm{X}}\right)$, electron harpooning takes place. The potential-energy surfaces for such important processes have been described in a classic series of papers by Berry, and the reader is referred to the review article which also describes many of the experiments done on these systems. ${ }^{25}$

In the femtosecond experiments by Rose et $a l^{4}$ and Rosker et al. ${ }^{5}$ the initial pulse at $\lambda_{1}$ takes the molecule from the $\mathrm{M}^{+} \mathrm{X}^{-}$ground state to the covalent surface $\mathrm{MX}^{*}$. The second pulse probes the reaction at $\lambda_{2}^{*}$ (transition-state region) and at $\lambda_{2}^{\infty}$ (final product; in this case $\mathrm{Na}$ ):

$$
\mathrm{MX}^{*} \rightarrow[\mathrm{M} \cdots \mathrm{X}]^{\ddagger *} \rightarrow \mathrm{M}+\mathrm{X} \quad(\mathrm{M}=\mathrm{Na} ; \mathrm{X}=\mathrm{I}, \mathrm{Br}) .
$$

En route to products, the $[\mathrm{M} \cdots \mathrm{X}]^{\ddagger *}$ transition-state molecules 'decide' between ionic and covalent paths; either the packet of $\mathrm{MX}^{*}$ will be trapped on the adiabatic potentialenergy curve or it will dissociate by following the non-adiabatic curve. The two cases have entirely different temporal behaviour and, if there is trapping, the frequency and amplitude of the oscillations will provide details of the nature of the surfaces and the strength of the coupling. Comparison with theory ${ }^{26}$ can then be made.

The experiments show (fig. 7) a striking oscillatory behaviour (when probing at $\lambda_{2}^{*}$ ) that persists for picoseconds ( $c a .10$ oscillations) in the case of $\mathrm{NaI}$, and for less time (ca. 2 oscillations) for the $\mathrm{NaBr}$ case. Probing on-resonance (at $\lambda_{2}^{\infty}$ ) gives a rise with plateaux separated by the same oscillation frequency. This on-resonance 'integration' of the trajectories is a clear demonstration of the concept of FTS, discussed earlier. The average period for $\mathrm{NaI}$ reaction is $1.25 \mathrm{ps}$, which corresponds to a frequency of $27 \mathrm{~cm}^{-1}$. It is concluded that the packet for $\mathrm{NaI}$ is effectively trapped in the adiabatic well en route to products, and that the observed oscillations represent 'pulses of $\mathrm{Na}$ atoms' leaving

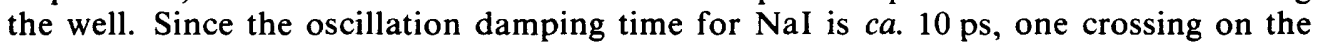



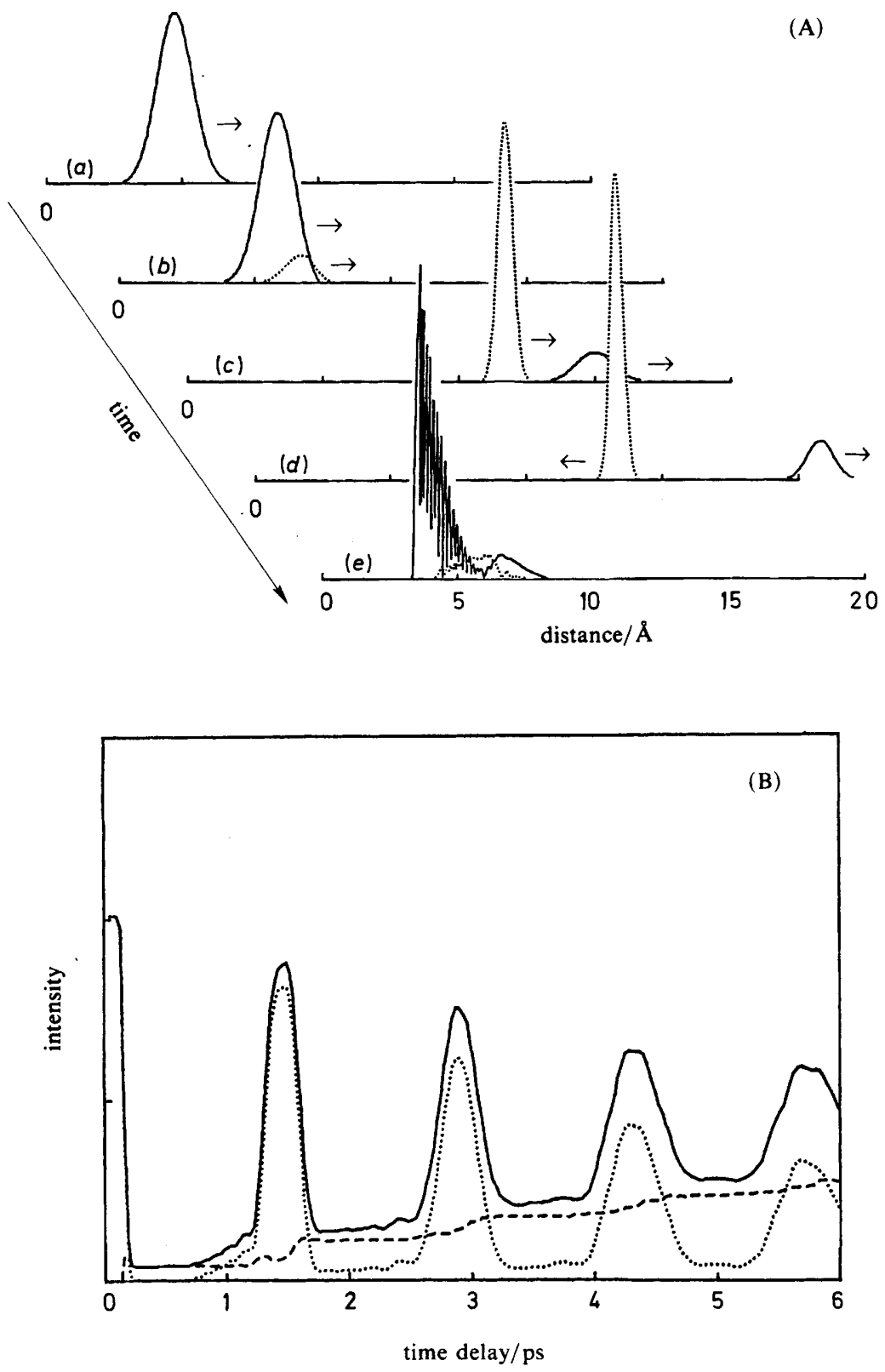

Fig. 8. (A) The quantum mechanical time evolution of the wavepacket in the reaction of NaI: (a) $160,(b) 200,(c) 500,(d) 700$ and (e) 1300 fs. Note the 'splitting' of the packet into ionic and neutral states and their separate time evolutions. These results are typical for the different $\lambda_{1}$ values studied. (B) The simulated FTS transients for $\lambda_{2}^{*}$ and $\lambda_{2}^{\infty}$ probings and the total signal (一). $\lambda_{1}=300 \mathrm{~nm}$. Note the similarity to the experimental results in fig. 7 . The calculations are detailed in ref. (27) by Engel et al. 
outward phase per oscillation ( $c a .1 \mathrm{ps)} \mathrm{has} \mathrm{a} \mathrm{probability} \mathrm{of} c a .0 .1$ to escape from the well. For $\mathrm{NaBr}$, the frequency of oscillation is similar in magnitude, but severe damping is observed. Thus, the crossing for $\mathrm{NaBr}$ is much more facile than for $\mathrm{NaI}$, consistent with theoretical expectation. In ref. (4) and (5) we compared theory with experiments and obtained the Landau-Zener probability of escape, the anharmonicity of the PES, and the coupling between the covalent and ionic curves. An interesting question is how the 'dephasing' and spread of the wavepacket influences the dissociation rate, as determined by the decay of the oscillations. The experiments do show manifestations of this decay and spreading, and theoretical modelling of the wavepacket dynamics in these prototype systems is important. Very recently, quantum and semiclassical calculations (fig. 8) have been performed ${ }^{27}$ and agreement with the experiment is good.

Real-time observations of the dynamics at different $\lambda_{1}$ and $\lambda_{2}^{*}$ should now allow one to view the motion of the wavepacket under different conditions from the Franck-Condon excitation region all the way to 'infinite separation,' or fragmentation, making contact with absorption spectroscopy, atomic beam scattering experiments and photofragment translational spectroscopy. Such experiments promise to provide rich information bearing upon the shape of the PES, curve crossings and interactions among different degrees of freedom in many other reactions. In future publications, ${ }^{28}$ we will detail these findings and relate to studies using wing emission spectra by Polanyi's group. ${ }^{29}$

\section{Femtosecond Alignment and the Angular Part of the PES}

To this point, we have discussed the reaction $\mathrm{ABC}^{*} \rightarrow \mathrm{A}+\mathrm{BC}$ as if its configuration space were one-dimensional (with no centrifugal contribution), the only parameter being the distance of A from the centre of mass of BC. However, the angular part of the potential may be important. As an example, the repulsion of bending $A B C$ will generate a torque during the reaction. This time-dependent evolution of angular momentum is related to the alignment, which can be studied using FTS by polarizing the fs pulses. Transients taken with parallel pump and probe polarizations can be compared with those taken with perpendicular polarizations, as done in time-integrated experiments, ${ }^{30}$ to probe the evolution on the femtosecond timescale.

The time dependence of alignment and angular momenta are reflected in two types of measurements. First, the angular part of the potential might lead to different $\tau_{1 / 2}$ values, depending on the final angular momentum state of the $\mathrm{BC}$ fragment. Secondly, the loss of alignment at early times will reflect the degree of the torque which leads to rotations in the $\mathrm{BC}$ fragment. Because the $\mathrm{BC}$ fragment most probably will be produced in a distribution of angular momentum states, the coherence time is expected to be short. These points are discussed next.

\section{(a) Time Evolution of Alignment and Coherence}

Here, we shall consider a triatomic molecule (e.g. ICN) with two coordinates, $R$ and $\theta$. $R$ is the distance between the I atom and the centre of mass of the CN. The angle between $r$ (the $\mathrm{CN}$ direction) and $R$ is $\theta$. Using the well known formula ${ }^{31}$ for the angular dependence of the signal, and transforming from the space-fixed coordinate system to the molecule-fixed axes system, one obtains expressions for measurements of the signal when the $E$ vector of the probing pulse is parallel, $I_{\|}(t)$, or perpendicular, $I_{\perp}(t)$, to the $E$ vector of the pump pulse:

$$
\begin{aligned}
& I_{\|}(t)=1+\frac{\beta}{10}+\frac{3 \beta}{10} \cos \left(2 j \omega_{0} t\right) \\
& I_{\perp}(t)=1-\frac{\beta}{20}-\frac{3 \beta}{20} \cos \left(2 j \omega_{0} t\right) .
\end{aligned}
$$


In these equations, an overall constant is neglected and $\omega_{0}$ is the fundamental rotational angular frequency. $j \hbar$ is the angular momentum of the fragment $(\mathrm{CN})$, and $\beta$, as usual, is equal to 2 for a parallel transition. (The $\mathrm{CN} \mathrm{B} \leftarrow \mathrm{X}$ transition is parallel.)

From the above expressions we note several points. First, in this case, the alignment of the $\mathrm{CN}$ (in a given $j$ ) generated by the dissociation impulse is periodic. Secondly, $I_{\|}(t)$ and $I_{\perp}(t)$ have identical periodicity but they are out of phase. The period of the oscillation is determined by $2 \omega_{0}$. At $t=0$ the ratio of the two absorptions is 3 . For the ICN dissociation, we can obtain the expected time evolution. Since $j \hbar=\omega I$, one obtains for $j=5$ that the $\mathrm{CN}$ will execute a revolution in $c a .1 .7 \mathrm{ps}$. For $j=25$, it will spend $c a$. $340 \mathrm{fs}$ in one revolution. $\dagger$ Accordingly, the transients will be periodic with the time constants determined by the characteristic $j$-state dynamics.

The problem is that the dissociation impulse does not produce the $\mathrm{CN}$ in one $j$ state. Instead, because of the distribution in the torque (in this case, the bending angles) many $j$ states are produced in the course of dissociation. For ICN, e.g. at $308 \mathrm{~nm}$ excitation, the average $C N$ rotational energy is $c a .1200 \mathrm{~cm}^{-1}$ (the average $j$ is 25 ) and the distribution is very wide. As a result of this distribution, the 'discrete coherence' described above will be replaced by 'dephasing,' and the question is then: what is the dephasing time, and what are the expected $I_{\|}(t)$ and $I_{\perp}(t)$ in the course of dissociation?

To handle these questions, we will assume a distribution of $j$ states produced by dissociation. For simplicity, we take this distribution, $P(j)$, to be a Gaussian (centred at $j=0$ ), and separate the alignment and population dynamics. Then, we can average the above equations over this distribution and obtain:

$$
\begin{aligned}
& \left\langle I_{\|}(t)\right\rangle=\left(1+\frac{\beta}{10}+\frac{3 \beta}{10} \exp \left(-t^{2} / \tau_{\mathrm{c}}^{2}\right)\right) A(t) \\
& \left\langle I_{\perp}(t)\right\rangle=\left(1-\frac{\beta}{20}-\frac{3 \beta}{20} \exp \left(-t^{2} / \tau_{\mathrm{c}}^{2}\right)\right) A(t)
\end{aligned}
$$

where $\tau_{\mathrm{c}}$ is the dephasing time given by $\left(\omega_{0} \Delta_{j}\right)^{-1}$, with $\Delta_{j}$ being the half-width of the distribution in $j . A(t)$ is the usual population ('incoherent') transient function (build-up of free fragment absorption or build-up and decay of transition-state absorption). We note that the ratio at $t=0$ is again 3 . However, the limiting values at long times will be 1.2/0.9 (and not 1) for exponential $A(t)$. The transients in this case will show decay in $I_{\|}$experiments and a build-up in the $I_{\perp}$ experiments with a time constant $\tau_{\mathrm{c}}$. This is reminiscent of the problem of rotational diffusion of molecules in liquids.

To see the connection, we write the expressions for the case of liquids: ${ }^{32}$

$$
\begin{aligned}
& I_{\|}^{\mathrm{L}}(t)=\frac{I_{0}}{3}[1+2 r(t)] \exp \left(-t / \tau_{\mathrm{f}}\right) \\
& I_{\perp}^{\mathrm{L}}(t)=\frac{I_{0}}{3}[1-r(t)] \exp \left(-t / \tau_{\mathrm{f}}\right)
\end{aligned}
$$

where $\tau_{\mathrm{f}}$ is the incoherent fluorescence decay, and $r(t)$ describes the rotational diffusion, usually given as $r_{0} \exp \left(-t / \tau_{\mathrm{r}}\right)$. According to these expressions, when $\tau_{\mathrm{f}}>\tau_{\mathrm{r}}$, then the ratio of $I_{\|}$and $I_{\perp}$ at $t=0$ is $\left(1+2 r_{0}\right) /\left(1-r_{0}\right)$. For a dipole transition, $r_{0}=0.4$ and this ratio is again 3 ! The signal will show the expected decay behaviour for $I_{\|}$and build-up for $I_{\perp}$, and as $t \rightarrow \infty$, the ratio is 1 (not 1.2/0.9). On the other hand, if $\tau_{\mathrm{f}}<\tau_{\mathrm{r}}$ then the ratio is simply $\left(1+2 r_{0}\right) /\left(1-r_{0}\right)$ at all times. The same kind of behaviour can be seen from eqn (7a) and (7b) in the case of dissociation if we take $A(t)$ to be an exponential decay, representing, e.g. the decay of the transition states.

\footnotetext{
† For simplicity of notation we shall use $j$ to denote the angular momentum of the $\mathrm{CN}$ fragment, although more conventionally we should use $N$.
} 


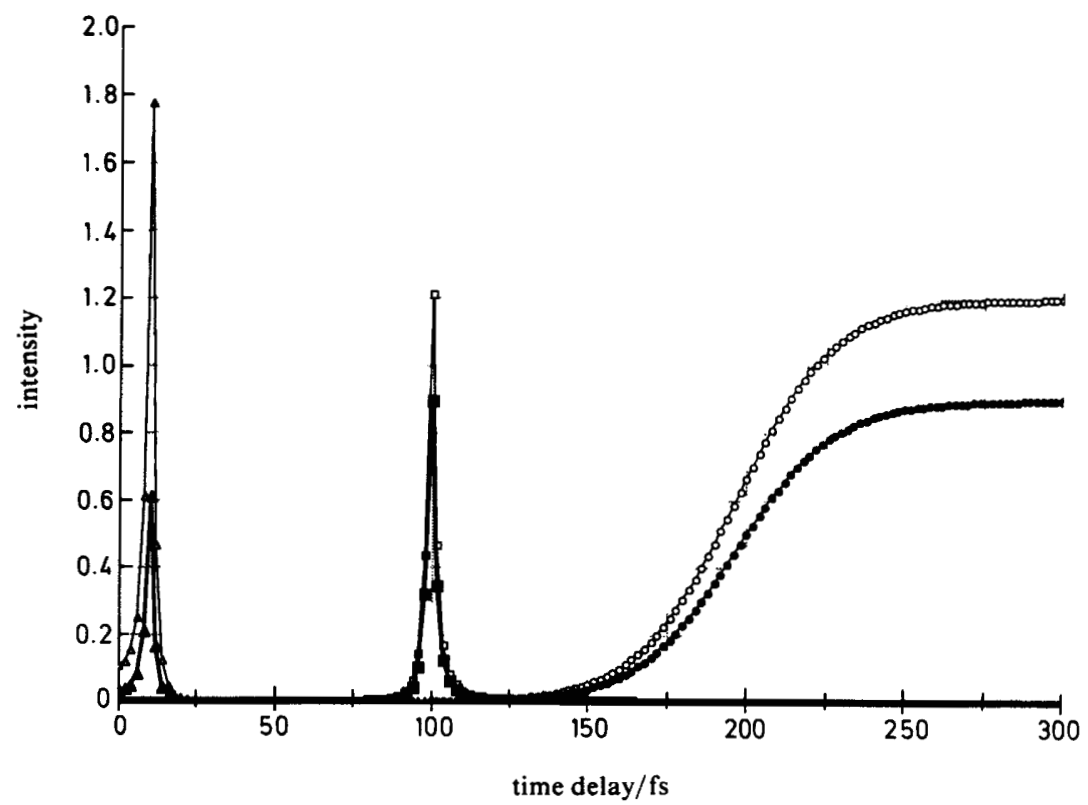

Fig. 9. The calculated $\left\langle I_{\|}(t)\right\rangle$ and $\left\langle I_{\perp}(t)\right\rangle$, showing the effect of $t^{*}(10 \mathrm{fs}, 100 \mathrm{fs}$ and $1 \mathrm{ps})$ and $\tau_{\mathrm{c}}$ ( $50 \mathrm{fs})$. The transients show the decrease of the anisotropy as $t^{*}$ increases. Note that the initial ratio is 3 , and at long time delays it becomes $1.2 / 0.9$. For longer $\tau_{\mathrm{c}}$ the transients are different (see text).

To compare theory [eqn (7)] with experiments, we take $A(t)$ to be the absorption of fragments during dissociation. The model for this $A(t)$ has been developed elsewhere, ${ }^{3,10}$ and here for illustration we consider the simplest case of a one-dimensional PES. Then,

$$
A(t)=\frac{C}{\gamma^{2}+W^{2}\left(t, t^{*}\right)}
$$

where

$$
W\left(t, t^{*}\right)=E\left(\operatorname{sech}^{2} x-\operatorname{sech}^{2} x^{*}\right) .
$$

The total energy is $E$ and $x$ and $x^{*}$ are simply $v t / 2 L$ and $v t^{*} / 2 L ; v$ is the recoil velocity, $L$ is the potential repulsion parameter [i.e. $L_{1}$ in eqn (3)], $t^{*}$ is the time for maximum absorption by the probe and $C$ is a constant. The model explains the observed transients when $t^{*} \rightarrow \infty$ (free fragment detection) and for finite $t^{*}$ values (transition states detection), and is consistent with quantum calculations as described above and shown in fig. 5 .

We now use eqn (7) and simulate the expected behaviour for $\left\langle I_{\|}\right\rangle$and $\left\langle I_{\perp}\right\rangle$ for different $\tau_{\mathrm{c}}$ and $t^{*}$. Fig. 9 shows these calculations. It is interesting to note that the anisotropy becomes more pronounced as $t^{*}$ becomes shorter. This is expected since the dephasing time is finite, and if we probe at early times on the PES we have a better chance for observing the large differences between $I_{\|}$and $I_{\perp}$. Note that if the rotational distribution of the fragment $P(j)$ is shifted from $j=0$, as in this case, then the coherence decay of eqn (7) will be modulated by $\cos \left(2 \omega_{0} j_{\max } t\right)$. For $j_{\max }=25$, the period of the oscillation is $170 \mathrm{fs}$, longer than $\tau_{\mathrm{c}}$, and hence is relatively unimportant. If the dephasing time is longer than $\tau_{1 / 2}$ then the effect of rotations will be seen in the post $200 \mathrm{fs}$ region of fig. 9. Naturally if the $\mathrm{BC}$ fragment is rotationally cold or the experiments detect only 
certain $j$, one will observe the beats [see eqn (6)] in this region, as described before. We will give details of these different limits elsewhere. ${ }^{14}$ Recently, quantum calculations on ICN by Metiu's group ${ }^{27}$ have shown this oscillatory behaviour, and comparison with these calculations will be made in ref. (14).

The dephasing time can be estimated for the ICN dissociation over the entire $j$-range. Since $\omega_{0}$ for the $C N$ is known and $\Delta j$ of the rotational distribution is also known, $\tau_{\mathrm{c}} \approx 40 \mathrm{fs}$. The calculation of this dephasing time due to an 'impulse' of dissociation has direct analogy to dephasing of molecules excited coherently into a distribution of rotational levels by a laser pulse. ${ }^{33}$ In this case one obtains an initial dephasing and then recurrences at long times (determined by the rotational constant of the molecule), as verified experimentally. ${ }^{33}$ If we apply this analogy to the problem at hand we obtain the following simple expression for $\tau_{\mathrm{c}}$ :

$$
\tau_{\mathrm{c}} \approx 2.2\left[B\left\langle E_{\mathrm{R}}\right\rangle\right]^{-1 / 2}
$$

where $\tau_{\mathrm{c}}$ is in ps and both the rotational constant $B$ and the average rotational energy $\left\langle E_{\mathrm{R}}\right\rangle$ are in $\mathrm{cm}^{-1}$. At $306 \mathrm{~nm}$ of pump excitation $\left\langle E_{\mathrm{R}}\right\rangle$ is known (see section 2) and this gives $\tau_{\mathrm{c}} \approx 44 \mathrm{fs}$ according to this model. The preliminary experimental data in fig. 10 give the $I_{\|}$and $I_{\perp}$ transients, and we will attempt an improvement of the $S / N$ and polarization extinction to permit extraction of an accurate $\tau_{\mathrm{c}}$. It is now clear that interesting new effects regarding temporal and spatial alignment can be searched for as we systematically change $t^{*}$, make the pulse duration even shorter and detect different $j$ states.

In the above treatment we did not consider the effect of parent rotation. This may be incorporated in $\beta$, but for the ICN it is known that parent rotation is not what determines the rotational distribution of the $\mathrm{CN}^{34}$ Finally, it will be interesting to compare the above theoretical calculations, based on the separation of coherent (alignment) and incoherent (population) dynamics, with 'exact' dynamical calculations on PESs of different angular forms. ${ }^{14}$ This will help us to understand the different timescales involved and their relevance to the separability question, since the $R$ and $\theta$ 'motions' are expected to be coupled.

\section{(b) Time Evolution of Angular Momenta}

The LIF spectrum of free CN exhibits the many transitions of the $P$ and $R$ branches. For $308 \mathrm{~nm}$ excitation, the $\mathrm{R}$ branch is fully resolved and extends to $381.5 \mathrm{~nm}$. These transitions to the blue of the bandhead are associated with different rotational quantum numbers of the $\mathrm{CN}$ fragment.

The spectral width of the femtosecond probe is broad, so that the excitation of a single rotational level is impossible. However, we have attempted to probe regions selectively within the $P$ branch of the $C N$ spectrum which are associated with either high or low rotational quantum states. For example, the region near the bandhead is associated with high rotational states $(\leqslant 20)$, and that near the start of the P branch at $387.5 \mathrm{~nm}$, with lower quantum numbers.

The clocking experiment was performed at various probe wavelengths between 388.7 and $387.4 \mathrm{~nm}$ The vaues of $\tau_{1 / 2}$ determined from these measurements ${ }^{3}$ are shown in fig. 11 for both 306 and $285 \mathrm{~nm}$ pump wavelengths. At the bandhead, as previously discussed, this experiment yields a time delay of $\tau_{1 / 2} \approx 200 \mathrm{fs}$. However, a substantial decrease in $\tau_{1 / 2}$ was observed as the probe was shifted towards shorter wavelengths.

An obvious possible cause for this observation is due to the change in the translational energy with rotational excitation of the $C N$. For $7000 \mathrm{~cm}^{-1}$ of total available energy, the low-angular-momentum states will be produced with essentially all this energy in translation. However, for high-angular-momentum states the translational energy will 

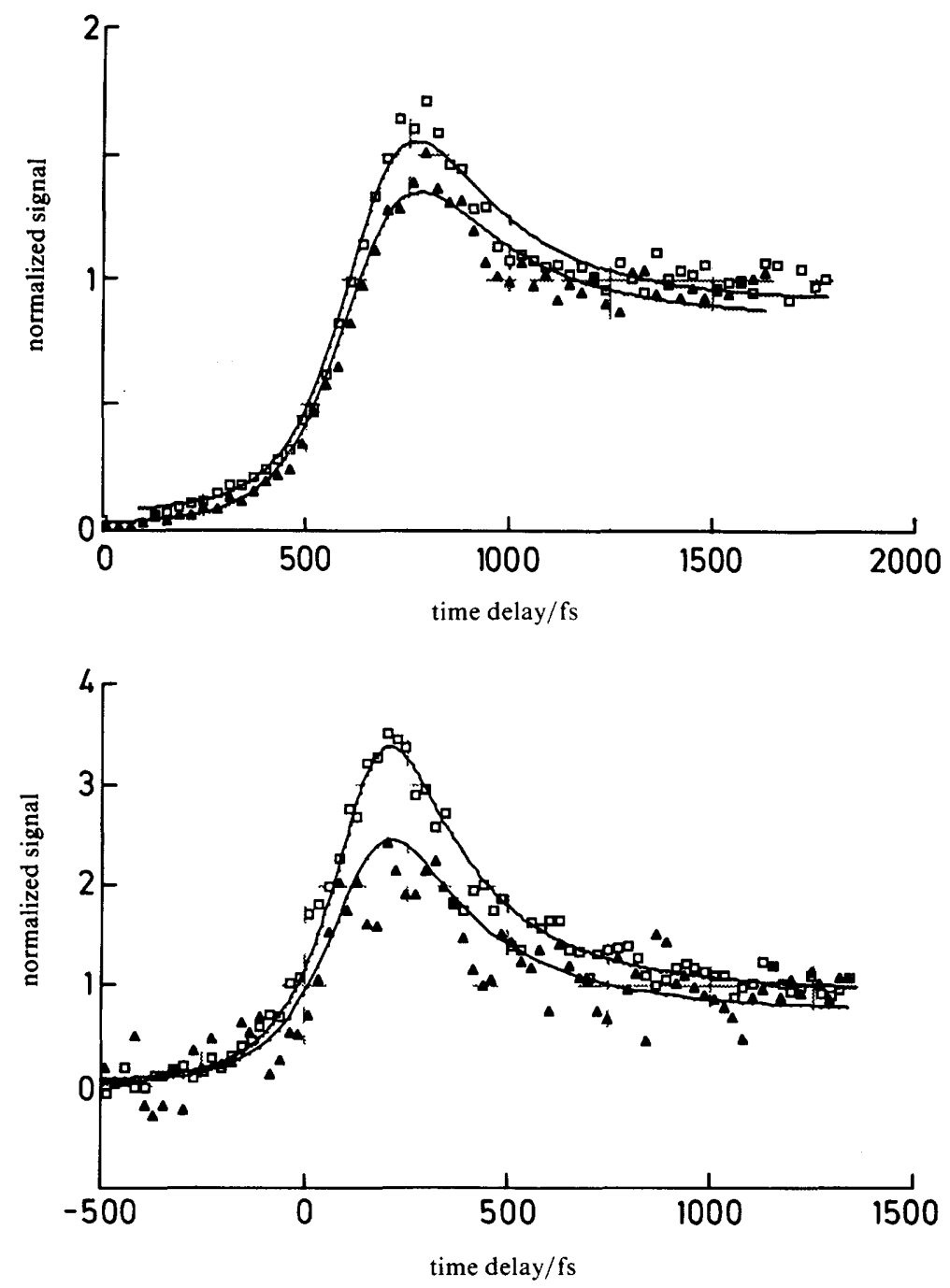

Fig. 10. Preliminary results of femtosecond alignment experiments. FTS data taken off-resonance, with either parallel $(\square)$ or perpendicularly polarized $(\boldsymbol{\Delta})$ pump and probe beams. The probe polarization was kept parallel to the detection axis. Two data sets are shown, taken under different experiental conditions. Note that the time delay in the upper curve is arbitrary (the zero-of-time was not determined for this set). The data are normalized to unity at long time delays. ${ }^{3}$ Experiments with better $S / N$ and polarization extinction are in progress.

be less than the available energy; for $j=25, E_{\mathrm{R}}=1250 \mathrm{~cm}^{-1}$, and the translational energy becomes $5750 \mathrm{~cm}^{-1}$ (vibrational excitation is assumed to be negligible). ${ }^{13}$ In the centreof-mass frame, $v$ is proportional to $E^{1 / 2}$, and $\tau_{1 / 2}$ at most should change from $c a .200$ to $c a .180 \mathrm{fs}$. This is a much smaller effect than the data in fig. 11 show.

Probing the different rotational states of the $\mathrm{CN}$ is sensitive to a more interesting dependence, namely, the angular part of the potential. In general, the PES is not only radial but it has an angular dependence described by the angle $(\theta)$ between $I$ and the centre-of-mass of the CN. For ICN, the potential has been postulated and used to fit the product state distribution, e.g. as done by Goldfield et al. ${ }^{9}$ A form that is discussed 


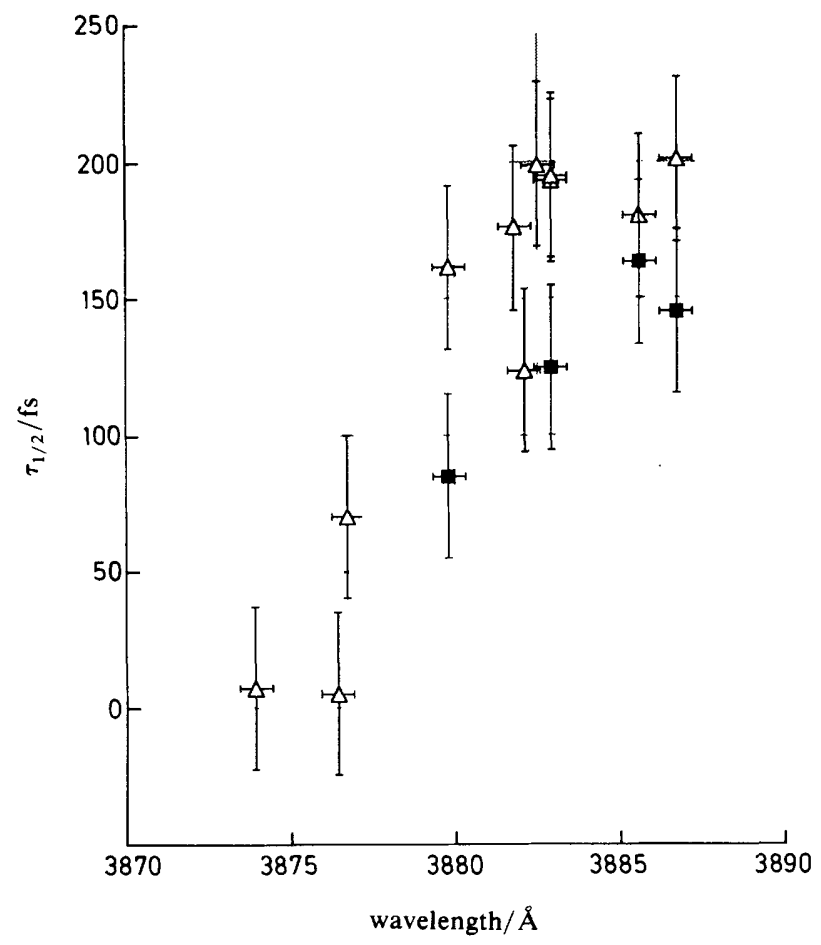

Fig. 11. Fragment delay time, $\tau_{1 / 2}$, as a function of probe wavelength. The values of $\tau_{1 / 2}$ obtained are shown for a pump wavelength of $c a .306 \mathrm{~nm}(\triangle)$ and $285 \mathrm{~nm}(\boldsymbol{\square})$. The f.w.h.m. of the probe was ca. $60 \mathrm{~cm}^{-1}$ in each case.

by Schinke and others is generally written as: ${ }^{35}$

$$
V(R, \theta)=A \exp \left(-\frac{R-R_{0}}{L}\right) \exp \left(-\frac{\varepsilon\left(\cos ^{2} \theta-1\right)}{L}\right)
$$

where $\varepsilon / L$ is a measure of the anisotropy of the potential. When $\varepsilon=0$, one recovers the single radial potential (isotropic). However, for negative $\varepsilon$, the potential describes a bent excited state which has a minimum at $\theta=90^{\circ}$ and a maximum at $\theta=0$. Dugan ${ }^{13}$ has discussed this bending (of CN relative to I) at the $308 \mathrm{~nm}$ excitation and obtained a bending angle of $\theta_{0}=18^{\circ}$. Basically, if the molecule were linear $\left(\theta_{0}=0\right.$, on the average), then the rotational distribution would show a maximum at $j \approx 0$; instead, the maximum seen in these experiments is at higher $j$ values for this wavelength of excitation. As he pointed out, at these long wavelengths there are one or two quanta of bending vibration (ground state), and their classical turning points are at 14 and $19^{\circ}$. We will not attempt to quantify the angle here, but the important points are that the bent state produces a range of torques and that there is time evolution of $j$.

From the potential, one can calculate the torque and hence $j(t)$ :

$$
\text { torque }=\mathrm{d} j / \mathrm{d} t=\boldsymbol{R} \times \boldsymbol{F}=\boldsymbol{R} \times[-\nabla \boldsymbol{V}(R, \theta)] .
$$

For illustration, we used Schinke's potential and calculated (using a simple torque) the evolution of $j(t)$. This is shown in fig. 12. The angular momenta evolve in very short, but finite times ( $c a .50 \mathrm{fs}$ or so) on the scale of femtochemistry experiments. The simple evolution shown here indicates that at shorter times the distribution is biased toward 


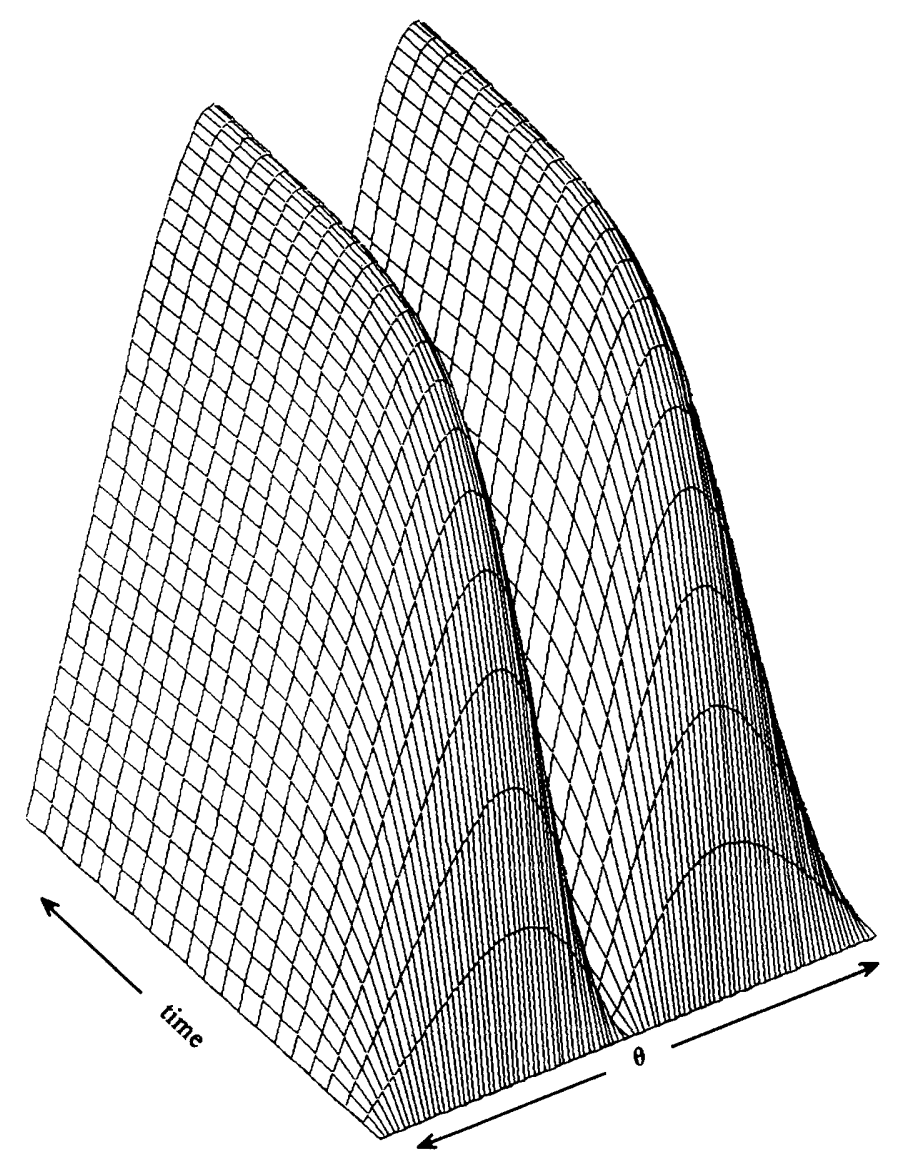

Fig. 12. Angular momentum as a function of time and angle, using a Schinke-type potential (see text) and $\varepsilon<0$. The time ( $10 \mathrm{fs}$ steps) is from 0 to $200 \mathrm{fs}$ and the angle is from $-\pi / 2$ to $+\pi / 2$. Note that when starting at the maxima and minima of the potential along $\theta(0, \pm \pi / 2, \ldots)$ no angular momentum is generated since $(\nabla V)_{\theta}=0$ and hence the torque is zero; see eqn (11) and (12). We have also calculated snapshots of the rotational distribution and found that at $10 \mathrm{fs}$, the distribution cuts off at $j \approx 10$, while for $55 \mathrm{fs}$ it cuts off at $j \approx 40$, assuming a simple torque.

lower $j$ states and these states evolve to their final $j$ values at longer times. We calculated snapshots of the rotational distribution at steps of $10 \mathrm{fs}$ and confirmed this picture.

It appears that probing different $\mathrm{CN}$ rotational states is monitoring different trajectories (reflecting the degree of the torque and the steepness of the PES along $\theta$ ) of different reaction times. To see this in real time and compare with experiments, we are currently examining trajectory calculations on PESs of different forms. For example, for the potential in fig. 13 (Goldfield et al. type ${ }^{9}$ ) we ran trajectories (fig. 14) and observed different times. We also observed more complex evolution for $j(t)$ than the one shown in fig. 12. (This is because, as mentioned above, we simplified the nature of the torque for the sake of illustration.) These calculations are in progress for different parameters and potentials to compare with experimental findings and examine the promise for probing the angular part of the dynamics. Much more will be learned in the coming years about these problems. We have considered here the simple cases but there are other possibilities and interpretations as yet unexplored. Hopefully as we gain more experience with these new findings we will be able to address these and other problems 


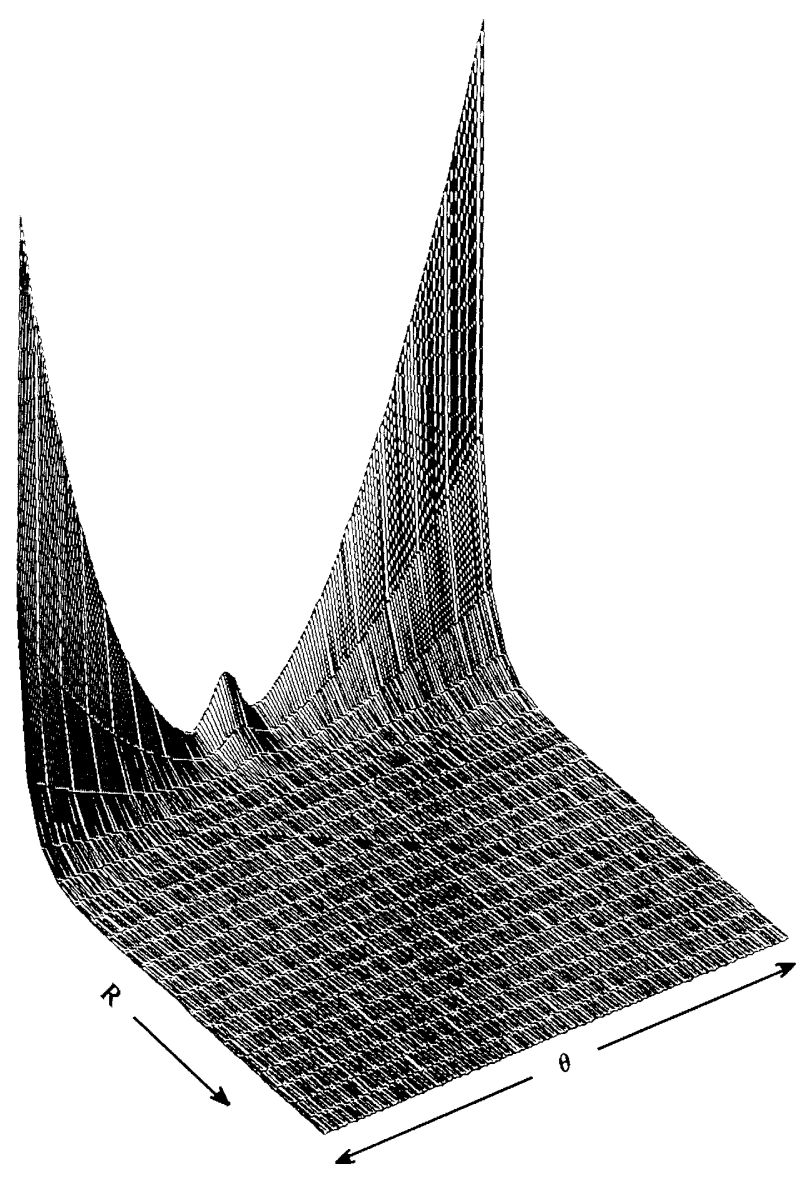

Fig. 13. A three-dimensional plot of a Goldfield et al-type PES (see text) for ICN. The angle $\theta$ is from $-\pi$ to $+\pi$ and $R$ is taken up to $6.7 \AA\left(R_{0}=2.7 \AA\right)$. The double well along $\theta$ describes the bent configurations.

of the PESs involved, like the effect of centrifugal barriers, van der Waals wells and crossings on $V_{1}$ and $V_{2}$ PESs.

\section{(c) Half-collision vs Full-collision}

The above discussions describe features of alignment, coherence and time evolution of angular momenta expected in reactions involving a half-collision. There are two points that should be made regarding other types of reactions which are bimolecular in nature, or exhibit resonance characteristics.

In cases where there is crossing (or avoided crossing) between different potentialenergy curves, as in the case of NaI, the temporal behaviour shown in fig. 7 suggests that alignment experiments should be of great interest. In this case, there is no torque, but the initial alignment of the parent should play a role depending on the time for the wavepacket oscillatory propagation and the rotation time of the parent molecule. Thus, probing at different $t^{*}$ should show the interplay between the two. For NaI, e.g., the rotation time of the molecule in the covalent excited state is $3.6 \mathrm{ps} \mathrm{rad}^{-1}$ for $j=30$. This means that during one crossing from the covalent to the ionic curve, the molecule rotates 


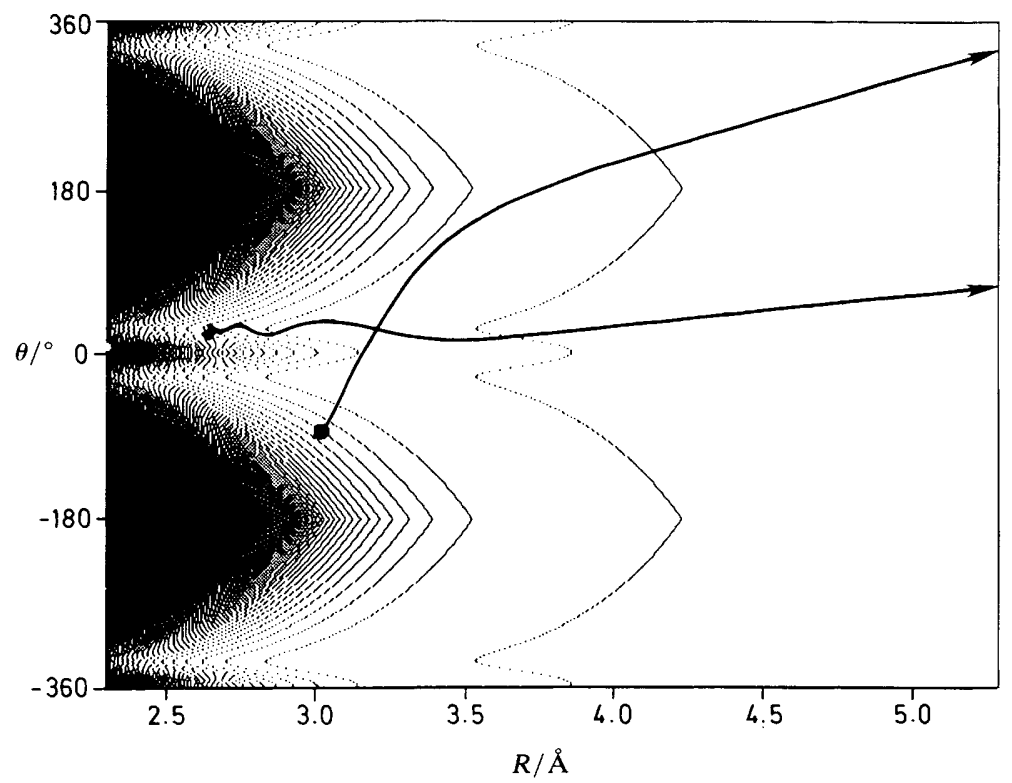

Fig. 14. A contour map of the PES of Goldfield et al. ${ }^{9}$ Using a computer algorithm for the classical dynamics, $\uparrow$ two trajectories on this surface are displayed. These calculations in fig. $12-14$, when completed, will be detailed in a separate publication.

an angle of $9.7^{\circ}$. Hence, measurements of $I_{\|}$and $I_{\perp}$ should show anisotropy that changes with the number of crossings. Such behaviour will be interesting to observe and relate to the dynamics of wavepacket dephasing.

In the other class of reactions discussed in this paper, namely 'oriented' bimolecular reactions, e.g. $\mathrm{H}+\mathrm{CO}_{2}$, these alignment experiments will be valuable. As shown ${ }^{36}$ by Herschbach many years ago, the mean time, $\tau_{\mathrm{d}}$, for the decomposition of the collision complex can be estimated from knowledge of the angular distribution (direct vs. complex mode reaction) and the rotational period of the complex,

$$
\tau_{\mathrm{r}}=2 \pi(I / L) \text {. }
$$

For example, for RbKCl, $I \approx 500 \mathrm{amu} \AA^{2}$ and for $L<500 \hbar, \tau_{\mathrm{r}}>1-2 \mathrm{ps}\left(\tau_{\mathrm{r}}\right.$ is still long compared to vibrational time). The symmetry in the angular distribution thus indicates that $\tau_{\mathrm{d}}$ is longer than $1-2$ ps for this class of reactions. $\dagger$

Real-time studies can now be made to obtain direct information on $\tau_{\mathrm{r}}$ and $\tau_{\mathrm{d}}$. If we monitor the collision complex (e.g. $\mathrm{HOCO}^{\ddagger}$ ) in real time and study the loss of the alignment then we will know $\tau_{\mathrm{r}}$ and, as discussed above, the time for the formation of the products can be measured directly to give $\tau_{\mathrm{d}}$. This will give the different timescales involved in the dynamics and allow one to examine the effect of limited impact parameter (stereospecificity) on the statistical or non-statistical behaviour of the reaction. There is a wealth of experiments to be performed in this direction to study the effect of available energy, orientation etc. As with our attempt to investigate the 'alkali age' reactions of $\mathrm{MX}$ in the femto age, we also plan to extend these types of experiments to the bimolecular femto age.

+ We are grateful to Prof. R. Bersohn for writing the program while visiting us at Caltech. The calculations in this figure were made by $\mathrm{Mr} \mathrm{H}$. Levy to obtain the trajectories, the $j(t)$ values and the rotational distributions. 


\section{Conclusions}

In this paper we presented some theoretical descriptions relating to the role of alignment and orientation in femtochemistry experiments. The experimental technique of femtosecond transition-state spectroscopy (FTS) is described and some applications to unimolecular, 'oriented' bimolecular, and alkali-metal halide reactions are presented. We did not detail other transition-state spectroscopies ${ }^{29,37-41}$ or reaction rate measurements, ${ }^{42,43}$ but these were discussed in ref. (1).

A key feature here is that because of the time resolution and sensitivity one is able to view the dynamics with a resolution that is sub-angstrom. ${ }^{1}$ With polarized femtosecond pulses an additional dimension can now be explored, and this allows one to probe both probe both the coherent (alignment) and incoherent (population) parts of the dynamics, as they evolve on the femtosecond timescale. The applications presented are just the beginning and the future is very promising, as we hope we and others will be able to study many other reactions in their transition-states, the 'during phase' of the reaction, ${ }^{44}$ and obtain characteristics of the PES in real time.

This work was supported by the AFOSR and the NSF. It is a pleasure to express my thanks to colleagues and members of my research group. In particular, the discussions I had with Professor R. Bersohn and Mr S. Baskin on the alignment problem and on eqn (6) and (10) were very illuminating. I also wish to thank M. Dantus, H. Levy, C. Sipes; T. Rose and M. Rosker for helping with some of the figures and calculations, and for stimulating discussions. I am grateful to Professor H. Metiu and Dr R. Heather for communicating their results of ref. (27) and for the discussion.

\section{References}

1 A. H. Zewail, Science, 1988, 242, 1645; and references therein to work on transition-state spectroscopy.

2 M. J. Rosker, M. Dantus and A. H. Zewail, Science, 1988, 241, 1200.

3 M. J. Rosker, M. Dantus and A. H. Zewail, J. Chem. Phys., 1988, 89, 6113; M. Dantus, M. J. Rosker and A. H. Zewail, J. Chem. Phys., 1988, 89, 6128.

4 T. S. Rose, M. J. Rosker and A. H. Zewail, J. Chem. Phys., 1988, 88, 6672.

5 M. J. Rosker, T. S. Rose and A. H. Zewail, Chem. Phys. Lett., 1988, 146, 175.

6 M. Dantus, M. J. Rosker and A. H. Zewail, J. Chem. Phys., 1987, 87, 2395.

7 N. F. Scherer, J. L. Knee, D. D. Smith and A. H. Zewail, J. Phys. Chem., 1985, 89, 5141.

$8 \mathrm{~J}$. Chem. Soc., Faraday Trans. 2, 1989, 85, issue 9, contains a number of papers concerning this subject.

9 E. M. Goldfield, P. L. Houston and G. S. Ezra, J. Chem. Phys., 1986, 84, 3120.

10 R. Bersohn and A. H. Zewail, Ber. Bunsenges. Phys. Chem., 1988, 92, 373.

11 M. A. O'Halloran, H. Joswig and R. N. Zare, J. Chem. Phys., 1987, 87, 303; and references therein.

12 I. Nadler, D. Mahgerefteh, H. Reisler and C. Wittig, J. Chem. Phys., 1985, 82, 3885.

13 C. H. Dugan and D. Anthony, J. Phys. Chem., 1987, 91, 3929; 1988, 92, 720; and references therein.

14 M. Dantus and A. H. Zewail, to be published.

15 R. B. Bernstein and A. H. Zewail, J. Chem. Phys., 1989, 90, 829.

16 S. O. Williams and D. G. Imre, J. Phys. Chem., in press.

17 N. F. Scherer, L. R. Khundkar, R. B. Bernstein and A. H. Zewail, J. Chem. Phys., 1987, 87, 1451;

N. F. Scherer, C. Sipes, R. B. Bernstein and A. H. Zewail, to be published.

18 G. Radhakrishnan, S. Buelow and C. Wittig, J. Chem. Phys., 1986, 84, 727.

19 C. Jouvet and B. Soep, J. Chem. Phys., 1984, 80, 2229.

20 G. Schatz, M. S. Fitzcharles and L. B. Harding, Faraday Discuss. Chem. Soc., 1987, 84, 359.

21 D. R. Herschbach, Le Prix Nobel, 1986 (Elsevier, Amsterdam, 1987).

22 Y. T. Lee, Science, 1987, 236, 793.

23 J. C. Polanyi, Science, 1987, 236, 680.

24 R. D. Levine and R. B. Bernstein, Molecular Reaction Dynamics and Chemical Reactivity (Oxford University Press, NY, 1987) and references therein.

25 R. S. Berry, in Alkali Haide Vapors, ed. P. Davidovits and D. L. McFadden (Academic Press, NY, 1979) and references therein.

26 R. Grice and D. R. Herschbach, Mol. Phys., 1974, 27, 159; S. A. Adelman and D. R. Herschbach, Mol. Phys., 1977, 33, 793. 
27 V. Engel, H. Metiu, R. Almeida, R. A. Marcus and A. H. Zewail, Chem. Phys. Lett., 1988, 152, 1; H. Metiu and $R$. Heather, personal communication.

28 T. S. Rose, M. J. Rosker and A. H. Zewail, to be published.

29 H. J. Foth, J. C. Polanyi and H. H. Telle, J. Phys. Chem., 1982, 86, 5027; see also ref. (1) for more discussion.

30 See the special issue on Dynamical Stereochemistry, J. Phys. Chem., 1987, 91. This issue has many excellent articles on the subject.

31 See e.g.: R. Bersohn, in Molecular Photodissociation Dynamics, ed. M. N. R. Ashford and J. E. Baggott (Royal Society Chemistry, London, 1987), p. 1.

32 See: K. B. Eisenthal, Acc. Chem. Res., 1975, 8, 118.

33 P. M. Felker and A. H. Zewail, J. Chem. Phys., 1987, 86, 2460; J. S. Baskin, P. M. Felker and A. H. Zewail, J. Chem. Phys., 1987, 86, 2483.

34 W. J. Marinelli, N. Sivakumar and P. L. Houston, J. Phys. Chem., 1984, 88, 6685.

35 R. Schinke, J. Phys. Chem., 1988, 92, 3195.

36 W. B. Miller, S. A. Safron and D. R. Herschbach, Discuss. Faraday Soc., 1967, 44, 108.

37 D. Imre, J. L. Kinsey, A. Sinha and J. Krenos, J. Phys. Chem., 1984, 88, 3956.

38 P. R. Brooks, R. F. Curl and T. C. Maguire, Ber. Bunsenges. Phys. Chem., 1982, 86, 401.

39 R. B. Metz, T. Kitsopoulos, A. Weaver and D. Neumark, J. Chem. Phys., 1988, 88, 1463.

40 J. C. Nieh and J. J. Valentini, Phys. Rev. Lett., 1988, 60, 519.

41 P. D. Kleiber, A. M. Lyyra, K. M. Sando, S. P. Heneghan and W. C. Stwalley, Phys. Rev. Lett., 1985, 54, 2003.

42 L. R. Khundkar, J. L. Knee and A. H. Zewail, J. Chem. Phys., 1987, 87, 77. N. F. Scherer and A. H. Zewail, J. Chem. Phys., 1987, 87, 97; J. L. Knee, L. R. Khundkar and A. H. Zewail, J. Chem. Phys., 1987, 87, 115.

43 For a recent review see: J. L. Knee and A. H. Zewail, Spectroscopy, 1988, 3, 44; and references therein. 44 1. W. M. Smith, Nature (London), 1987, 328, 760.

Paper 8/03587C; Received 15th September, 1988 